\title{
A Rule-Based Verification and Control Framework in Atlas Trigger-DAQ
}

\author{
A. Kazarov, A. Corso-Radu, G. Lehmann Miotto, J. E. Sloper, and Yu. Ryabov
}

\begin{abstract}
In order to meet the requirements of ATLAS experiment data taking, the Trigger-DAQ (TDAQ) system is composed of $\mathrm{O}(\mathbf{1 0 0 0 0 )}$ of applications running on more than 2600 computers in a network. With such a system size, software and hardware failures are quite frequent. To minimize system downtime, the Trigger-DAQ control system shall include advance verification and diagnostics facilities. The operator shall use tests and expertise of the TDAQ and detectors developers in order to diagnose and recover from errors, if possible automatically.

The TDAQ control system is built as a distributed tree of controllers, where the behavior of each controller is defined in a rulebased language allowing easy customization. The control system also includes a verification framework which allows users to develop and configure tests for any component in the system with different levels of complexity. It can be used as a stand-alone test facility for a small detector installation, as part of the general TDAQ initialization procedure, and for diagnosing problems which may occur during run time.

The system is currently being used in TDAQ commissioning at the ATLAS experimental zone and by subdetectors for stand-alone verification of the detector hardware before it is finally installed.

Index Terms-Artificial intelligence, command and control systems, control systems, data acquisition, diagnostic expert systems, distributed computing, distributed control, distributed information systems, expert system shells, expert systems, intelligent control, large-scale systems, process control, programmable control, system analysis and design.
\end{abstract}

\section{INTRODUCTION}

A TLAS (A Toroidal LHC ApparatuS) [1] is a general-purpose high-energy physics experiment on the Large Hadron Collider being constructed in CERN.

The ATLAS Trigger and Data Acquisition (TDAQ) system [2] is a distributed system that performs filtering and transferring of the ATLAS experimental data from the detector to the mass storage. TDAQ system is steered by a TDAQ Control system that guarantees its coherent and smooth functioning.

The paper describes some core components of the ATLAS TDAQ Control system. The key feature of these components is that they are based on the expert-system technology so the behavior of the system is described in a rule-based language. This technique should help the TDAQ operator to minimize system

Manuscript received August 25, 2006; revised April 4, 2007.

A. Kazarov is with CERN, 1211 Geneva 23, Switzerland, on leave from St. Petersburg NPI, RU-188 350 Gatchina, Russian Federation.

A. Corso-Radu and G. Lehmann Miotto are with CERN, 1211 Geneva 23, Switzerland.

J. E. Sloper is with CERN, 1211 Geneva 23, Switzerland, on leave from University of Warwick, Coventry CV4 7AL, U.K.

Yu. Ryabov is with St. Petersburg NPI, RU-188 350 Gatchina, Russian Federation.

Digital Object Identifier 10.1109/TNS.2007.897825 down time and to control it smoothly by using the expertise of the system developers and to allow to adopt to the changing conditions of the experiment.

In the paper we describe the motivation, the design principles, the architecture and some details of the implementation of Run Control, Setup and DVS (Diagnostic and Verification System) components. The DVS component is described in more details including examples of its usage for ATLAS commissioning.

\section{Design AND ARChitecture OF THE RULE-BASED CONTROL FRAMEWORK}

\section{A. Motivation and Objectives}

ATLAS Trigger-DAQ system is a distributed computing system that transfers and filters data from the detector front-end electronics to the mass-storage for offline analysis. It is composed of a large number of hardware and software components, as summarized below:

- 1800 read-out VME boards;

- 1800 fiber links;

- 150 Read-Out System PCs each hosting 4 ROB-IN cards;

- 500 Level-2 Trigger PCs;

- 90 Sub-Farm Interface PCs;

- 2000 Event Filter PCs;

- 30 Sub-Farm Output PCs;

- 50 infrastructure PCs (file servers);

- 200 Ethernet switches;

- and $\mathrm{O}(10000)$ applications running on the listed above hardware.

The TDAQ Control system shall be capable of controlling this number of various components, meeting many requirements [2]. The size of the system under control and the number of components make the probability of a failure very high. The typical failures one faces every day are computer component failures and application crashes. Keeping in mind the life-time of the experiment (around 10 years) and the high cost of its down-time and as well the fact that the system will be operated by a non-expert shift crew, it is very important to have system verification, failure diagnostics and automated recovery facilities embedded in the control system of the TDAQ. These facilities should allow the TDAQ operator to:

- Detect problems as early as possible by means of regular testing of the system.

- Make use of the expertise (knowledge) of system's developers in order to diagnose problems.

- Automate verification of a large system.

- Minimize system down-time using (automated when possible) recovery procedures based on problem diagnosis. 


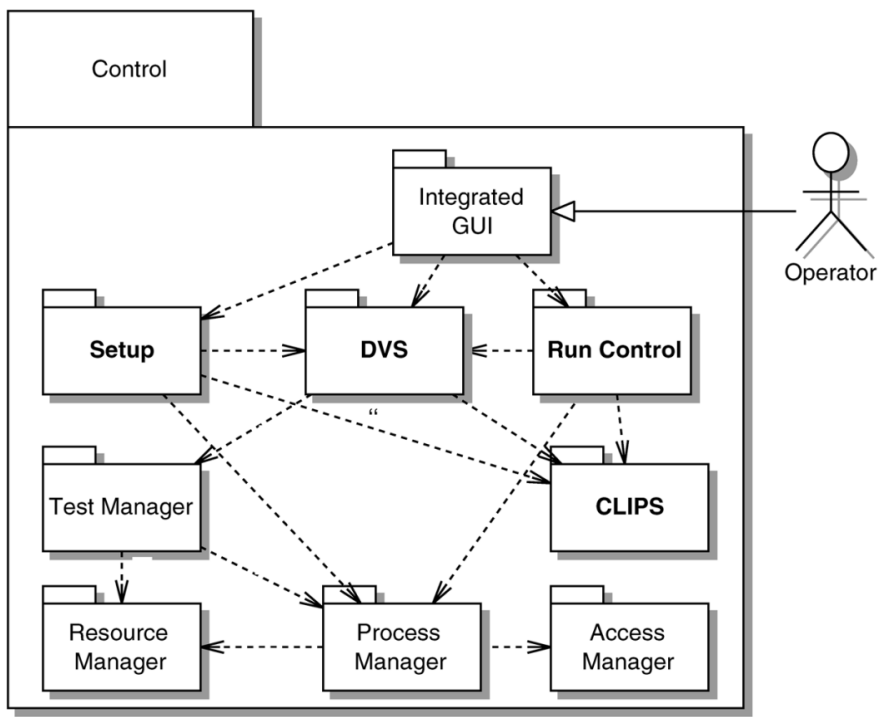

Fig. 1. Architecture of the Control subsystem.

Another important aspect of the TDAQ system which has an important influence on the design, is the geographical decentralization of the development of the experiment and a big number of users participating in the system development all over the world. In order to build the full-scale TDAQ system its software will be used by all ATLAS subdetectors each providing specific functionality, whereas the 'core' software should join all pieces together and guarantee their coherent functioning. This brings the idea of the 'core' framework components providing dedicated services and well-defined interfaces for users and developers and allowing customization and extensibility.

\section{B. Design Principles}

To fulfil the requirements it was decided to design the Control system following these principles:

- Framework approach: a system shall be configurable and extensible by the experts and users during development stage and also during the life-time of the experiment.

- Rule-based expert system approach: the system's behaviour is described in a rule-based language to allow accumulation of expert's knowledge and an easy adaptation to changing conditions. An expert system shell is used as the core of the control components.

- Hierarchical and distributed architecture approach: the composition of the Control system shall reflect the tree-like structure and the scale of the experiment. The distributed architecture will also guarantee that the scale and complexity of the system (i.e., the number of objects and rules that expert system deals with) does not affect its performance.

\section{Architecture of the Control Subsystem}

On the UML diagram below (Fig. 1) the actual architecture of the ATLAS Control subsystem is presented.

It is composed of a number of packages or components which implement a particular functionality and interact with other components via well-defined public interfaces. In this paper we briefly describe Run Control, DVS and Setup components which are the high-level building blocks of the Control subsystem. These components shares expert system technology provided by the CLIPS package which is also described. Other important packages directly used by the high-level components are Test Manager, Resource Manager, Process Manager and Access Manager providing required pieces of functionality, e.g., to start and supervise processes in distributed environment or to control access to the shared system resources. These low-level components are not covered in this paper.

Both Setup and Run Control components use the verification facilities provided by the DVS framework in order to verify the functionality of a subset of the system under control.

The TDAQ Operator interacts with the system via a humanfriendly Integrated Graphical User Interface which runs on the control desktop.

The Control subsystem interacts tightly with other TDAQ subsystems, especially with Configuration and Monitoring ones (not shown on the figure for simplicity). The Configuration subsystem stores TDAQ configuration data for a particular run in a database and provides access to it for all other subsystems. The Monitoring subsystem implements services like message reporting and information sharing in a distributed environment.

In the following sections we describe in more details the main components of the Control subsystem: Run Control, Setup, DVS and CLIPS. We start from the CLIPS system which is the core technology for all main components.

\section{CLIPS: AN EXPERT SySTEM SHELL}

The behavior of the Run Control components which are mostly data- and event-driven is described naturally in terms of rules, rather than in a procedural language. It is also necessary to have an easy way to update the knowledge about the behavior of the system during the experiment lifetime, saving expert knowledge and detailed information about recovery procedures applied by the experts. Therefore it was decided to use the rule-based expert system technology as the base for all core Run Control components.

Some evaluations [3] have been done, and the CLIPS (stands for 'C'-Language Integrated Production System) expert system shell [5], [6] was selected. It was originally developed by NASA. It is free, open and well documented. The system is implemented in ' $\mathrm{C}$ ' programming language and provides all functionality in 'C' API, therefore it can be easily integrated with other software products.

CLIPS implements a traditional rule-base programming paradigm where rules and facts can be defined. In addition, a powerful feature of CLIPS is the object-oriented language ' $\mathrm{COOL}$ ' which allows to define classes, methods and instances and to define rules for the classes. Also, the conventional procedural programming constructs like functions, cycles and conditional jumps are available.

CLIPS is an expert system shell, i.e., this is an engine to build and run expert system applications. A particular application built on top of the shell becomes an expert system when it is populated with a knowledge base (KB), in terms of rules, classes and objects. In CLIPS, the rules and classes are described in human-readable and editable form in plain text files which are 


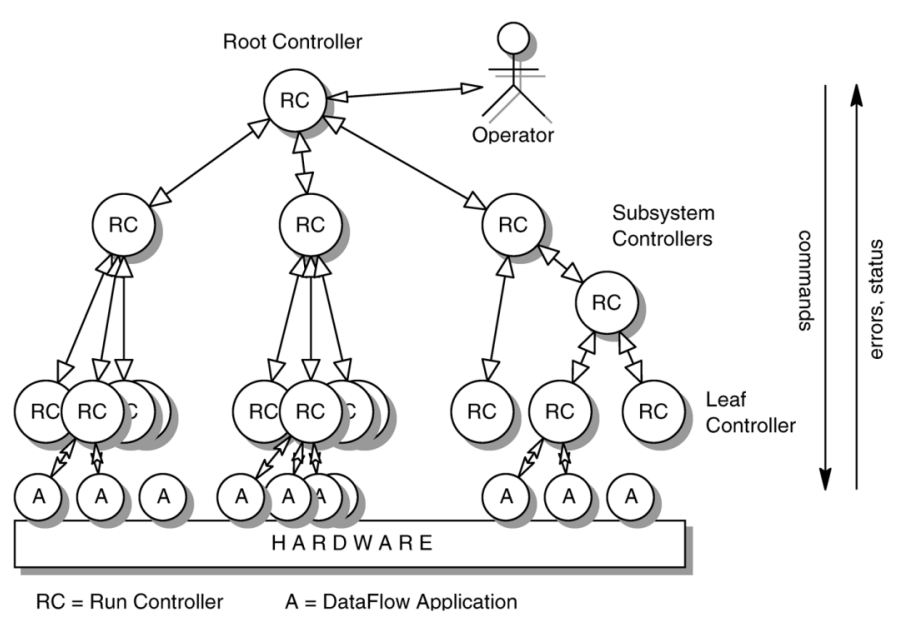

Fig. 2. Tree of Run Controllers.

loaded by the CLIPS engine. The set of objects is loaded into CLIPS memory from the TDAQ Configuration service for each particular configuration of the TDAQ system.

The main Control components are in fact CLIPS-based applications, each loading particular set of rules and interacting with other TDAQ components to fulfill its functionality.

\section{RUN CONTROL}

Run Control is a distributed framework allowing each subsystem in TDAQ to define the behavior of a particular controller according to a pre-defined state machine skeleton (see [3]) and to join all controllers in a distributed tree structure, in order to guarantee synchronous and homogeneous execution of Operator commands through the whole system. The top-level Run Controller reports the status of the system to the Operator and accepts his commands, while leaf controllers deal with applications which directly control the TDAQ hardware. Intermediate controllers represent statuses of different subsets of TDAQ.

Each controller is responsible of:

- distributing commands to its children;

- receiving children's statuses and analysing (diagnosing) error messages;

- determining its own state;

- executing of tests defined for components under control;

- undertaking possible recovery actions;

- passing status and error information to its parent.

The general picture of the Run Control tree is presented in Fig. 2.

Each controller is an application running on some machine in the system. The controller implements a pre-defined Finite State Machine skeleton (common for all controllers), defining its all possible states and transitions, and a small expert system (i.e., an expert system engine and a set of rules or a Knowledge Base, KB) defining its behavior. The details of the implementation are described in [3]. Rules are loaded from text files and each controller in the system may have specific set of rules loaded. The default rules included in the framework implementation are very simple, like: "if all my children controllers are in state 'A',

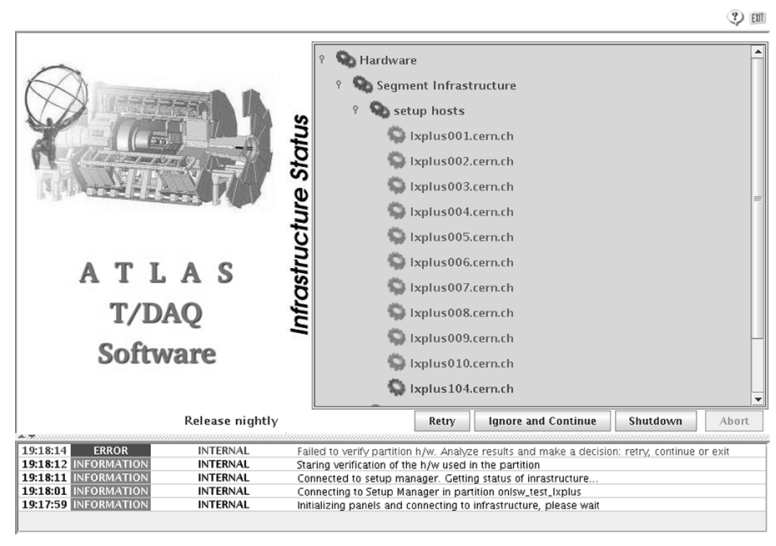

Fig. 3. Setup GUI window with hardware infrastructure being tested.

then change my state to 'A", or "if one of my children is in 'error' state (e.g., timed-out in transition, reported an error or became unavailable), then change my state to 'error' "'

More sophisticated recovery rules should analyze all available data and messages and make decisions like disabling a sub-tree of the system, executing pre-defined recovery actions or reporting an unrecoverable error to the parent controller, so that it could deal with the problem.

The concrete recovery policies are to be defined when the final system will be put into operation and may evolve during the experiment run-time, so the framework should allow easy adaptation of controller's behavior.

\section{SETUP}

The Setup component is a 'boot-strap controller' for the initial infrastructure of a particular TDAQ configuration. It brings the system to a state in which it can accept commands from Run Control system. It uses DVS to verify in depth the system hardware used in the configuration for the current run. Specific tests and rules developed by particular subsystems are executed in order to detect potential problems in advance and to confirm the system's integrity before launching any process. If some tests fail the user is provided with options like 'ignore and continue', 'retry' or 'abort'. Automatic recovery rules may also be defined.

The Setup KB contains additional rules to start, restart and verify applications and diagnose related problems. Most of the infrastructure applications have back-up capabilities, so they are restarted automatically if they crash or even if the host machine stops running. In the latter case a back-up host is chosen and applications are restarted there.

The functionality of applications under supervision is also confirmed by the execution of tests. This allows to check the status of an application from functional point of view, not relying only on the fact that the process is running. An application is started only if the hardware it uses is in a good state and all other applications it depends on have passed testing.

In Fig. 3 the initial GUI window of the TDAQ software is shown. On the right side one can see the Infrastructure panel with the tree of hardware components (a rack of PCs in this case) being tested, where one PC (lxplus104.cern.ch) did not pass testing, attracting the Operator attention. 


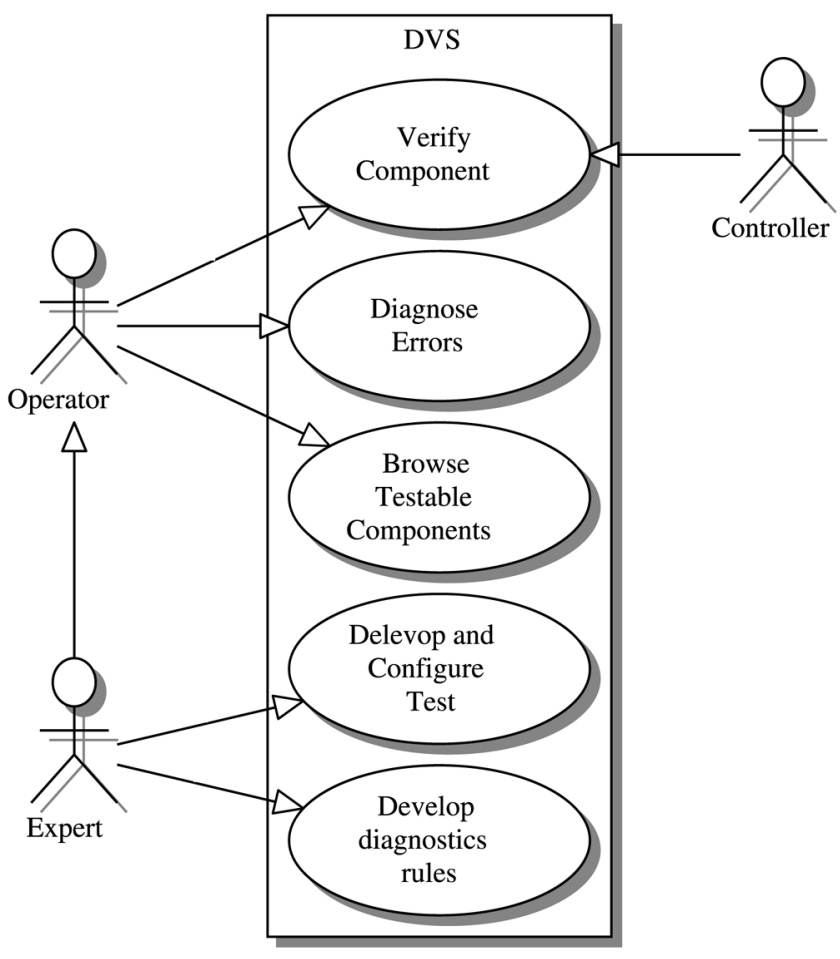

Fig. 4. Use Cases for DVS.

\section{Diagnostics AND VERIFICATION SYSTEM}

Diagnostic and Verification System (DVS) is an expert-system based framework which allows a user to:

- Define and configure a test for any component in the system.

- Have a testable view on the particular TDAQ configuration in a user-friendly GUI.

- Automate testing of the system.

- Make a diagnostics conclusion in case of a problem detected during testing (provided some knowledge has been put into the Knowledge Base)

- Extend diagnostics facilities by defining more specific rules for particular tests and components.

\section{A. Use Cases}

In Fig. 4 use cases for the DVS framework are presented. First, a TDAQ Expert (typically a developer of the system) contributes to the framework by developing specific tests and providing some diagnostic knowledge. Afterwards, a TDAQ Operator can reuse Expert's expertise for verifying any subset of the system and diagnosing detected errors. The verification functionality is also used by non-human bodies like Run Controller applications in order to check the functionality of the subsystem under control.

\section{B. Architecture}

The architecture of the DVS framework is shown in Fig. 5. Its core components are the Test Repository database describing all tests, and the expert system shell filled with some knowledge by an expert. The DVS functionality is available to end-users via $\mathrm{C}++$ and Java API that allows to embed the DVS functionality

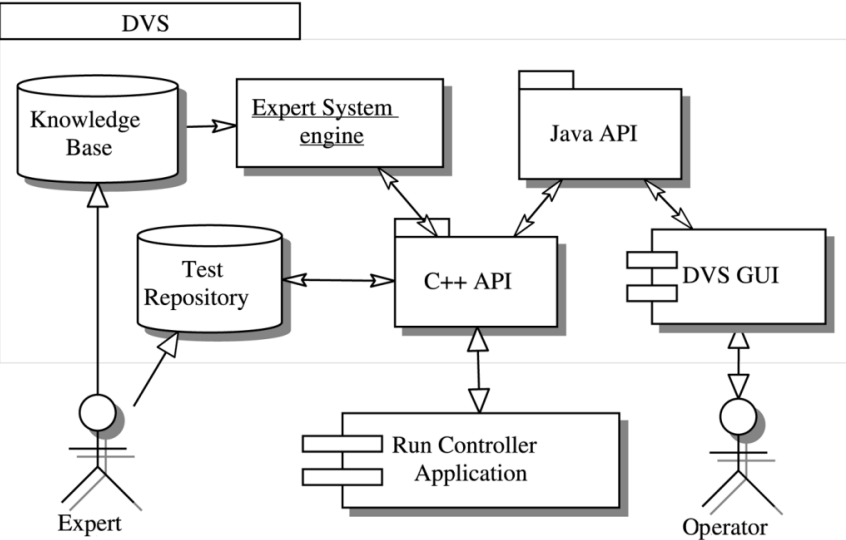

Fig. 5. DVS architecture.

in other applications (e.g., Run Controller application), and also as a stand-alone Java-based GUI application.

\section{Tests Configuration}

A test is a binary running on a particular host in a system. It verifies a specific functionality of a TDAQ component and returns a single result value: PASSED, FAILED, UNRESOLVED, TIMEOUT, and some additional text output. For a single component a number of different tests can be associated which can be organized in sequences, executed synchronously or asynchronously. Tests and their relationships are fully described in a database. The following attributes are available for the description of a test: command-line parameters, host, dependencies, timeouts, scope, complexity levels, the test mask and the mode of execution.

Any test can be associated either to a particular object in the configuration database or to all objects of a particular class. In the latter case a special template syntax can be used in test's parameters and host, so the real values are calculated when the test instance is launched for an object.

The mode of test execution has special meaning when it is specified for a test for a composite object, i.e., for an object which has subcomponents. It allows to execute tests for all children of this object either in parallel (if 'asynchronous' mode is selected) or sequentially (in case of 'synchronous' mode).

Tests levels and masks are used for more precise test selections which allow to promptly configure the test repository without editing the database. The user can select a subset of tests with a particular level of complexity or only those tests which names match some text pattern (test mask).

Test scope can be specified to prevent conflicting tests from being executed when the system is taking data.

Usually test is a non-interactive program which does not require interaction with the user. This allows to execute many tests in parallel and to test the whole system in one go. However interactive tests, called 'actions', are also available to allow users to execute more complex test scenarios requiring some input. This also allows to reuse already existing console utilities in the framework. An action is configured as a normal test, but it is launched in a separate terminal window where user can type in. 


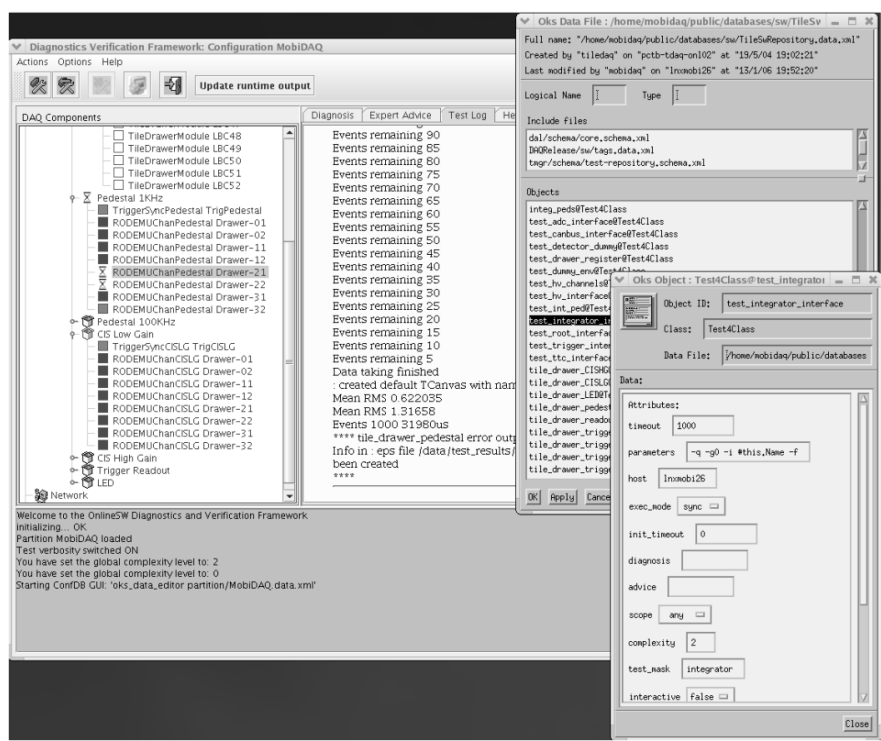

Fig. 6. DVS GUI with running tests and MobiDAQ test repository in configuration DB.

In Fig. 6 on the right side, two database editor windows are shown, one with the list of tests defined in the configuration and another one with description of a particular test.

\section{GUI}

In Fig. 6 one can see DVS GUI in action (the big window in the background).

On the left side of the window the tree of testable components is displayed. The user can browse the configuration and select a single component or a group in the tree and then to run all defined tests by clicking a single button in the toolbar. From the menu, user can select different parameters of testing, like test complexity and test verbosity and make test selection by applying a mask on the test name. The component icons change color reflecting the result of testing. User can follow the testing progress interactively without need to wait until testing of all components completed.

On the right side the test results are presented in dedicated panels. It includes test standard output and also diagnosis made by the expert system. The level of verbosity of tests can be defined globally in the menu. Test output produced by all children components can be combined in one panel and then saved in a file.

User can see output of a test as it executes in real time, even before it finishes. This is important for long running tests.

\section{E. Use of DVS for ATLAS Commissioning}

DVS is currently used by different ATLAS subdetectors and TDAQ subsystems in the ATLAS commissioning activities. A number of tests were developed for different detector and DAQ components, so they can be extensively tested before being installed at the final location.

One good example is 'MobiDAQ' system developed by the Tile calorimeter group [7]. This is a stand-alone mobile system which is used to automate testing of the Tile detector hardware. It is composed of a VME crate which controls the detector front-end electronics and a PC running TDAQ software including DVS which is used to execute sets of tests to verify different aspects of the detector functionality, like integrity of cable connections and the quality of signal propagation. Different tests for detector front-end electronics and for ROD (Read-Out Driver) VME modules were developed. The tests are executed in parallel for independent sections of the detector, and combined tests output is saves as a report in a file. In Fig. 6 on the left side you can see DVS GUI with a number of specific detector tests being executed. On the right side two configuration database editor windows are displayed, one with the list of all defined tests and another with the configuration of 'test_integrator_interface' test.

\section{CONCLUSION}

The paper describes three major components of the ATLAS TDAQ Control framework: setup, Run Control and DVS. These components share the rule-based expert system functionality, which allows to store and reuse expert knowledge during system run time in order to automatize system verification, problem diagnosis and recovery and therefore to minimize the system downtime. Such functionality is very important for the smooth steering of the large scale data taking system of the ATLAS experiment.

The approach to use expert system technique for the failure diagnostics and recovery of a complex system was already exploited in some previous high-energy physic experiments [8], but the scale of the present experiment makes important impact on the TDAQ control system architecture: it is made hierarchical, distributed and extensible.

The components are currently used for ATLAS subdetectors and TDAQ system commissioning.

\section{REFERENCES}

[1] ATLAS Tech. Proposal. Geneva, Switzerland, 1994, ATLAS Collaboration, CERN/LHHCC/94-43, LHCC/P2, CERN.

[2] B. Gorini et al., "The ATLAS data acquisition and high-level trigger: Concept, design and status," in Proc. Computing in High Energy Physics Conf., Mumbai, India, Feb. 2006.

[3] D. Liko et al., "Control in the ATLAS TDAQ system," in Proc. Computing in High Energy Physics Conf., Interlaken, Switzerland, Sep. 2004.

[4] A. Kazarov et al., "Verification and diagnostics framework in ATLAS trigger/DAQ," in Proc. Computing in High Energy and Nuclear Physics Conf., La Jolla, CA, Mar. 2003.

[5] CLIPS Expert System Shell documentation. [Online]. Available: http:// www.ghg.net/clips/download/documentation/

[6] J. C. Giarratano and G. D. Riley, "Expert systems: Principles and programming, fourth edition: Principles and programming," Course Technol., Oct. 15, 2004, ISBN-13: 978-0534384470.

[7] MobiDAQ web page [Online]. Available: http://atlas.web. cern.ch/Atlas/SUB_DETECTORS/TILE/Commissioning/mobidaq/HowTo.htm

[8] P. Mato, "DEXPERT: An expert system for read-out error recovery in the aleph data acquisition system," in Proc. 2nd Int. Workshop Software Engineering, Artificial Intelligence and Expert Systems in High Energy and Nuclear Physics, L'Agelonde, France, Jan. 13-18, 1992, pp. 537-542. 